\title{
Spin flipping a stored polarized proton beam with an rf dipole
}

\author{
V. A. Anferov, B. B. Blinov, D. Yu. Kantsyrev,* A. D. Krisch, and D. W. Sivers ${ }^{\dagger}$ \\ Randall Laboratory of Physics, University of Michigan, Ann Arbor, Michigan 48109-1120 \\ C. M. Chu, P. Schwandt, and B. von Przewoski \\ Indiana University Cyclotron Facility, Bloomington, Indiana 47408-0768 \\ V. N. Grishin \\ IHEP, Protvino 142 284, Russia \\ (Received 17 November 1999; published 24 April 2000)
}

\begin{abstract}
We recently studied the spin flipping of a $202.7 \mathrm{MeV}$ vertically polarized proton beam stored in the Indiana University Cyclotron Facility cooler ring during the first polarized run with its new cooler injector synchrotron and its new cooler injector polarized ion source. We first set the vertical betatron tune to avoid the measured $\nu_{y}$ value of the $G \gamma=7-\nu_{y}$ intrinsic depolarizing resonance in the cooler ring. We then flipped the spin by ramping the frequency of an rf dipole through an rf-induced depolarizing resonance. After optimizing the rf dipole's frequency ramp parameters, we used multiple spin flips to measure a maximum spin-flip efficiency of $97.5 \pm 1 \%$.
\end{abstract}

PACS numbers: 29.27.Bd, 29.27.Hj, 41.75.Ak

There is a growing interest in polarized beam experiments in storage rings such as the Indiana University Cyclotron Facility (IUCF) cooler ring [1], the MIT-Bates storage ring [2], the Brookhaven relativistic heavy ion collider (RHIC) [3], and HERA at DESY [4]. Frequent reversals of the beam polarization direction can significantly reduce the systematic errors in an experiment's spin asymmetry measurements. An rf solenoid was used earlier [5] to spin flip a vertically polarized proton beam stored in the cooler ring with no Siberian snake [6]. Since a solenoid's spin rotation decreases linearly with energy, a solenoid is impractical for spin flipping in high energy rings. However, a dipole's spin rotation is energy independent. Therefore, we recently used an rf dipole to spin flip a $202.7 \mathrm{MeV}$ vertically polarized proton beam stored in the IUCF cooler ring with no Siberian snake.

In any flat circular accelerator or storage ring with no horizontal magnetic fields, each proton's spin precesses around the vertical fields of the ring's dipole magnets. The spin tune $\nu_{s}$, which is the number of spin precessions during one turn around the ring, is proportional to the proton's energy

$$
\nu_{s}=G \gamma,
$$

where $G=(g-2) / 2=1.792847$ is the proton's gyromagnetic anomaly and $\gamma$ is its Lorentz energy factor.

This vertical spin precession can be perturbed by the horizontal rf magnetic field from either an rf solenoid or an $\mathrm{rf}$ dipole. This perturbation can induce an $\mathrm{rf}$ depolarizing resonance, which can be used to flip the

\footnotetext{
*Also at: Moscow State University, Moscow, Russia.

${ }^{\dagger}$ Also at: Portland Physics Institute, Portland, OR 97201.
}

spin direction of the ring's stored polarized protons [5,7]. The frequency $f_{r}$, at which an rf-induced depolarizing resonance occurs, is given by

$$
f_{r}=f_{c}(k \pm G \gamma),
$$

where $f_{c}$ is the proton's circulation frequency and $k$ is an integer. Sweeping the rf magnet's frequency through $f_{r}$ can flip the spin. The Froissart-Stora equation [8] relates the beam's polarization after crossing the resonance $P_{f}$ to its initial polarization $P_{i}$,

$$
P_{f}=P_{i}\left\{2 \exp \left[\frac{-\left(\pi \varepsilon f_{c}\right)^{2}}{\Delta f / \Delta t}\right]-1\right\},
$$

where $\varepsilon$ is the resonance strength and $\Delta f / \Delta t$ is the resonance crossing rate, while $\Delta f$ is the frequency range during the ramp time $\Delta t$.

The apparatus used for this experiment, including the rf dipole [9], the IUCF cooler ring, and the polarimeter, were discussed earlier [5,10-22] and are shown in Fig. 1. The $202.7 \mathrm{MeV}$ vertically polarized proton beam in the cooler ring was obtained using the new cooler injector polarized ion source (CIPIOS) [23] and the new cooler injection synchrotron (CIS) [24]. For this first polarized run with CIS and CIPIOS, the beam polarization was $75 \pm 2 \%$ after the $7 \mathrm{MeV}$ linac. However, the cooler ring's measured beam polarization was only about $20 \%$ because the $G \gamma=2$ imperfection resonance in CIS was not yet corrected [25].

The spin tune $\nu_{s}$ in the cooler ring was almost [12] equal to $G \gamma$, which is 2.18017 at $202.7 \mathrm{MeV}$; the beam circulation frequency $f_{c}$ was $1.96580 \mathrm{MHz}$. Thus, there should be an rf depolarizing resonance with its frequency near

$$
f_{r}=f_{c}\left(3-\nu_{s}\right)=1.6080 \mathrm{MHz} .
$$




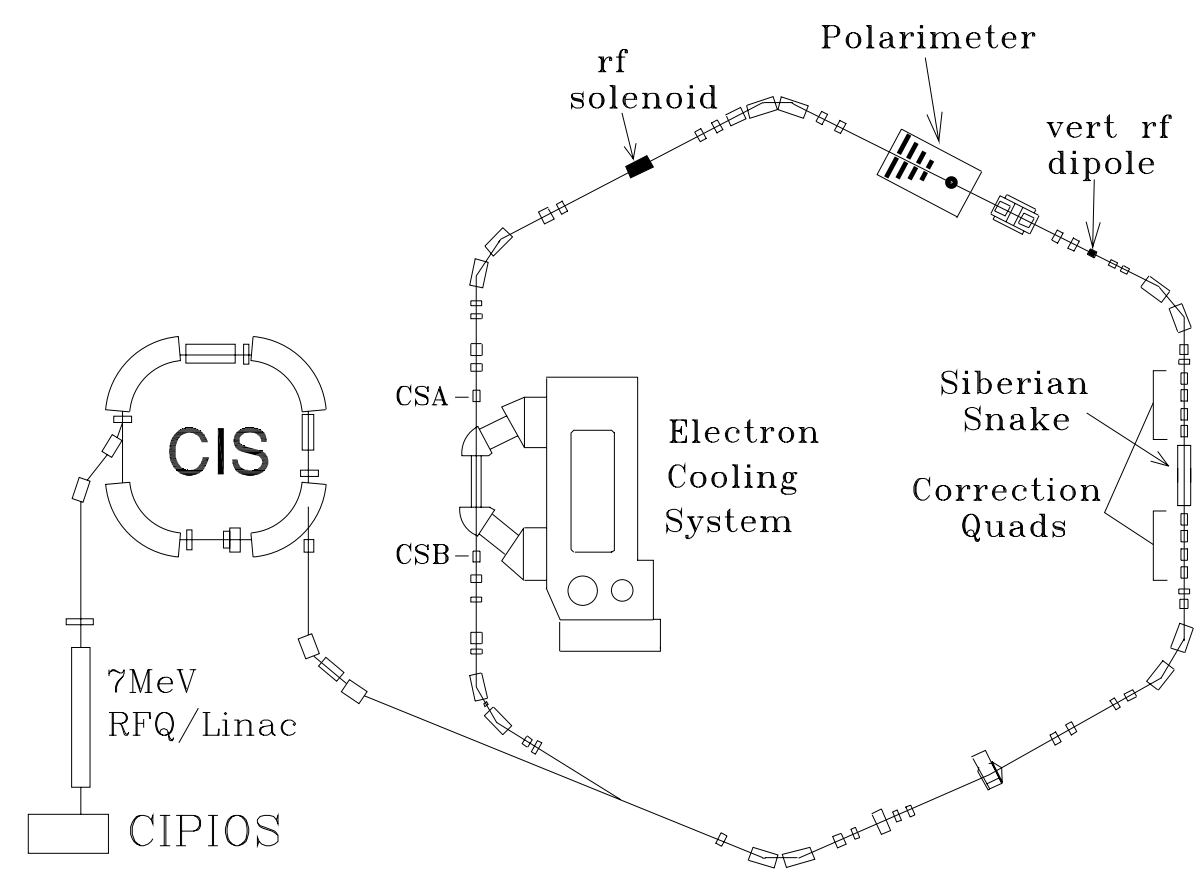

FIG. 1. Layout of the IUCF cooler ring with its new CIS and its new CIPIOS polarized ion source. Also shown in the cooler ring are the rf dipole, the polarimeter, and the Siberian snake which was off.

We excited this rf depolarizing resonance by operating the rf solenoid or the rf dipole near $1.6080 \mathrm{MHz}$. An intrinsic depolarizing resonance can also occur if the vertical betatron tune $\nu_{y}$ is set near the resonance value

$$
\nu_{y}=7-\nu_{s}=4.818 \text {. }
$$

We first varied the vertical betatron tune to determine the location of the $7-\nu_{y}$ intrinsic depolarizing resonance. The measured vertical beam polarization is plotted against the betatron tune in Fig. 2. As expected [11], the depolarization dip was observed near $\nu_{y}=4.817$.

For the rf-dipole-resonance spin-flipping studies, we set the vertical betatron tune at 4.791, which was well away from the intrinsic resonance. The rf dipole's input voltage was set at $47 \mathrm{~V} \mathrm{rms}$, corresponding to an $\int B d l$ amplitude of $0.06 \mathrm{~T} \mathrm{~mm}$. The vertical polarization was then measured at different dipole frequencies; this measured vertical polarization is plotted against the $\mathrm{rf}$ dipole's frequency in Fig. 3. The curve is a second-order Lorentzian fit to the data with a resonance frequency $f_{r}$ of $1607600 \pm 55 \mathrm{~Hz}$ and a width $w$ of $1630 \pm 100 \mathrm{~Hz}$.

To study spin flipping, we crossed this rf-induced resonance by linearly ramping the rf dipole's frequency, through the measured $f_{r}$, from $f_{r}-2$ to $f_{r}+2 \mathrm{kHz}$, with various ramp times $\Delta t$, while measuring the beam polarization after each frequency ramp. The measured vertical polarization is plotted against the ramp time in Fig. 4. Note that, after a very rapid increase, the polarization's magnitude seems to decrease slightly for ramp times above $100 \mathrm{msec}$. By comparing the measured polarization of $17.9 \pm 1.2 \%$ at $\Delta t=0$ with the value of $16.4 \pm 1.0 \%$, which is the average of the three data points in the $\Delta t$ range of 100 to $150 \mathrm{msec}$, we obtained a $92 \pm 8 \%$ spin-flip efficiency.

To further optimize the spin-flipping efficiency, we next varied the rf dipole's frequency range $\Delta f$ with its ramp time $\Delta t$ set at $80 \mathrm{msec}$, its amplitude set at $0.06 \mathrm{~T} \mathrm{~mm}$, and its range centered around $1607.7 \mathrm{kHz}$. The data indicated that the spin-flip efficiency had a maximum near $\Delta f=6 \mathrm{kHz}$.

After setting $\Delta t$ and $\Delta f$ to optimize the spin-flip efficiency, we more precisely determined this efficiency by

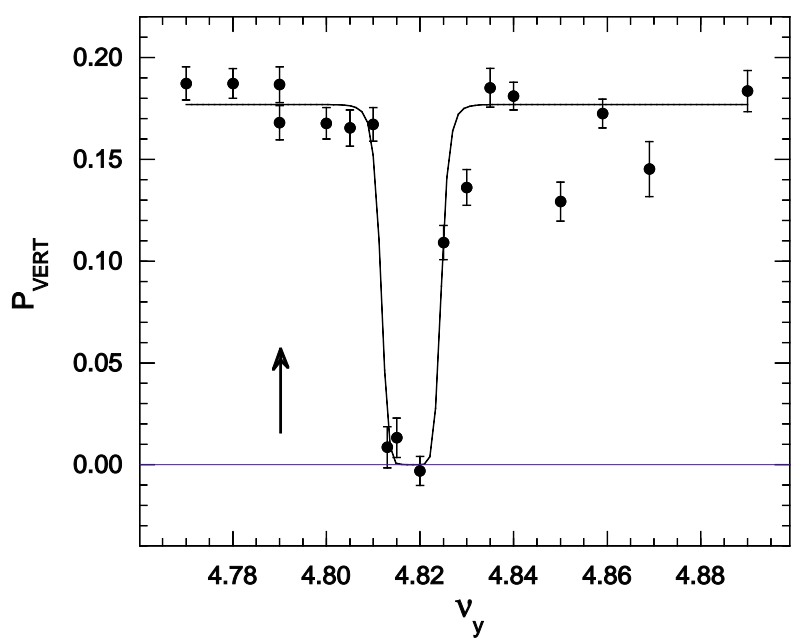

FIG. 2. (Color) The measured vertical proton polarization at 202.7 MeV is plotted against the vertical betatron tune. The curve is hand drawn to guide the eye. For the later studies, we set $\nu_{y}$ at the arrow value of 4.791, which is well away from the $\nu_{s}=7-\nu_{y}$ resonance value. 


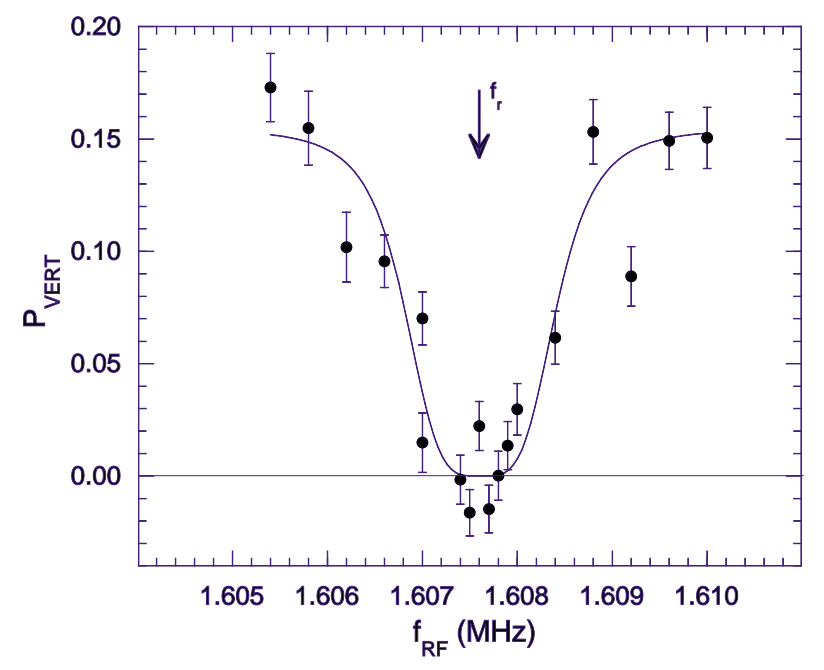

FIG. 3. (Color) The measured vertical proton polarization at 202.7 MeV is plotted against the rf dipole's frequency. The rf dipole's $\int B d l$ was $0.06 \mathrm{~T} \mathrm{~mm}$. The curve is a fit using a second-order Lorentzian.

measuring the vertical polarization after many spin flips. We varied the number of spin flips while keeping, for each spin flip, the ramp time, the frequency range, and the rf voltage all fixed. The vertical polarization is plotted against the number of spin flips in Fig. 5. We fit this data using

$$
P=P_{0} \epsilon^{n},
$$

where $P_{0}$ is the initial polarization, $\epsilon$ is the spin-flip efficiency, and $n$ is the number of spin flips. The best fit to the optimized spin-flip efficiency is $97.5 \pm 1 \%$.

In summary, we used an rf dipole to spin flip a stored 202.7 MeV vertically polarized proton beam with no Siberian snake in the IUCF cooler ring. We reached

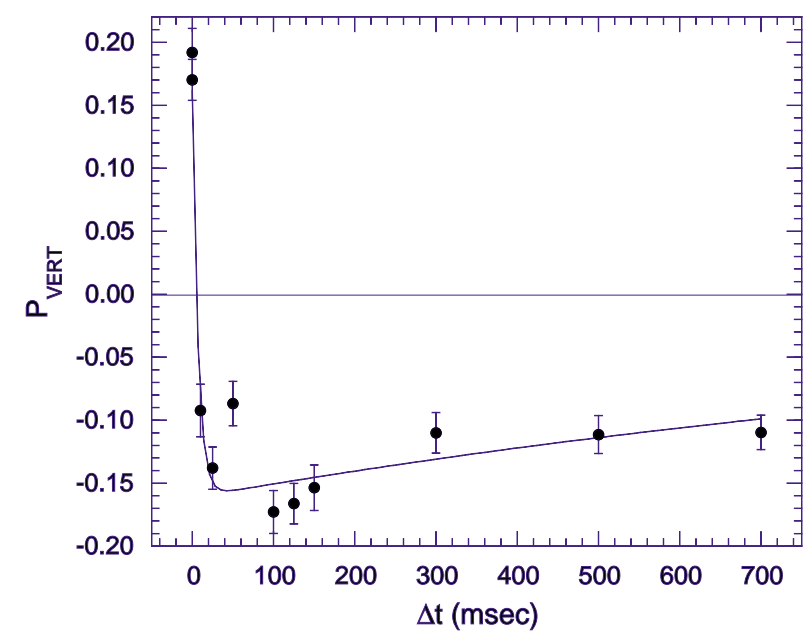

FIG. 4. (Color) The measured vertical proton polarization at 202.7 $\mathrm{MeV}$ is plotted against the rf dipole ramp time $\Delta t$. The frequency range $\Delta f$ was $4 \mathrm{kHz}$ and the rf dipole's $\int B d l$ was $0.06 \mathrm{~T} \mathrm{~mm}$. The curve is hand drawn to guide the eye.

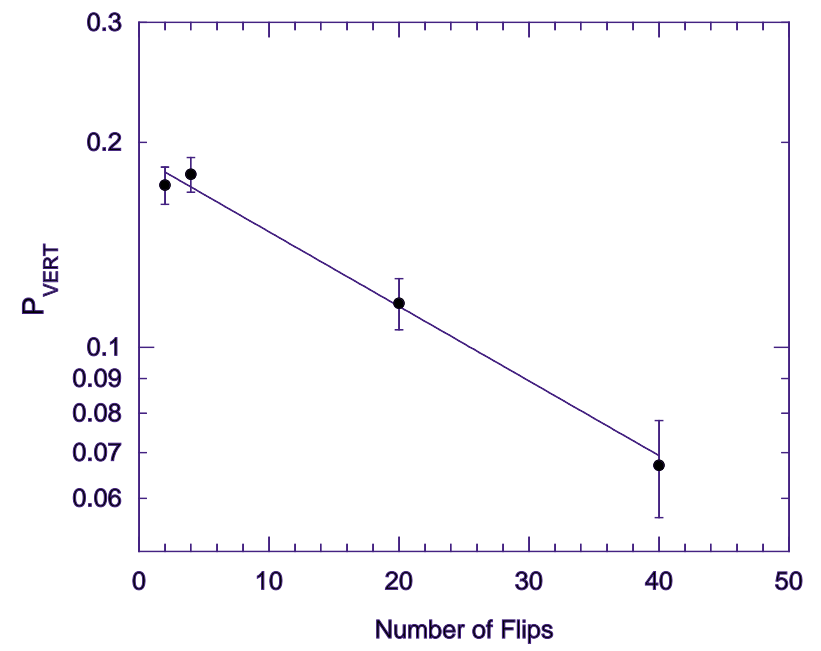

FIG. 5. (Color) The measured vertical proton polarization at 202.7 MeV is plotted against the number of spin flips. The rf dipole's ramp time $\Delta t$ was $80 \mathrm{msec}$, its frequency range $\Delta f$ was $6 \mathrm{kHz}$, and its $\int B d l$ was $0.06 \mathrm{Tmm}$. The curve is a fit to the data using Eq. (6).

a measured spin-flip efficiency of $97.5 \pm 1 \%$; it may have been reduced by possible nearby weak higher-order resonances [20]. Our future plans include increasing the spin-flip efficiency with both an $\mathrm{rf}$ dipole and an $\mathrm{rf}$ solenoid, both with and without a Siberian snake in the ring, developing a spin-flipping technique for an exactly $100 \%$ Siberian snake, and further studying higher-order snake resonances.

\section{ACKNOWLEDGMENTS}

We would like to thank J.M. Cameron, A.S. Belov, V.P. Derenchuk, G. W. East, D. L. Friesel, T. Rinckel, W. T. Sloan, E. J. Stephenson, and the entire Indiana University Cyclotron Facility staff for the successful first polarized operation of the cooler ring with its new CIS and CIPIOS polarized ion source. We are grateful to R. Baiod, A. W. Chao, E. D. Courant, T. Kageya, F.Z. Khiari, S. Y. Lee, A. M. T. Lin, W. Lorenzon, P. S. Martin, H. O. Meyer, M. G. Minty, C. Ohmori, R. A. Phelps, R. E. Pollock, L. G. Ratner, T. Roser, A. D. Russell, H. Sato, T. Toyama, and V.K. Wong for their help with earlier parts of this experiment and to B. R. Rubin for his help in running. This research was supported by grants from the U.S. Department of Energy and the U.S. National Science Foundation.

[1] H. O. Meyer et al., Phys. Rev. Lett. 81, 3096 (1998); B. von Przewoski et al., Phys. Rev. C 58, 1897 (1998).

[2] BLAST Collaboration, R. Alarcon et al., Bates Report No. 2-39, 1999.

[3] Y. Makdisi, in High Energy Spin Physics: Eleventh International Symposium, edited by K. J. Heller and S. L. 
Smith, AIP Conf. Proc. No. 343 (AIP, New York, 1995), p. 75.

[4] SPIN Collaboration, University of Michigan Report No. UM-HE 96-20, 1996; UM-HE 99-05, 1999.

[5] D. D. Caussyn et al., Phys. Rev. Lett. 73, 2857 (1994).

[6] Ya.S. Derbenev and A. M. Kondratenko, Part. Accel. 8, 115 (1978).

[7] B. W. Montague, Phys. Rep. 113, 35 (1984).

[8] M. Froissart and R. Stora, Nucl. Instrum. Methods 7, 297 (1960).

[9] D. A. Crandell et al., Phys. Rev. Lett. 77, 1763 (1996).

[10] A. D. Krisch et al., Phys. Rev. Lett. 63, 1137 (1989).

[11] J. E. Goodwin et al., Phys. Rev. Lett. 64, 2779 (1990).

[12] M. G. Minty et al., Phys. Rev. D 44, R1361 (1991).

[13] V. A. Anferov et al., Phys. Rev. A 46, R7383 (1992).

[14] R. Baiod et al., Phys. Rev. Lett. 70, 2557 (1993).

[15] R. A. Phelps et al., Phys. Rev. Lett. 72, 1479 (1994).

[16] B. B. Blinov et al., Phys. Rev. Lett. 73, 1621 (1994).
[17] C. Ohmori et al., Phys. Rev. Lett. 75, 1931 (1995).

[18] L. V. Alexeeva et al., Phys. Rev. Lett. 76, 2714 (1996).

[19] R. A. Phelps et al., Phys. Rev. Lett. 78, 2772 (1997).

[20] C. M. Chu et al., Phys. Rev. E 58, 4973 (1998).

[21] B. B. Blinov et al., Phys. Rev. Lett. 81, 2906 (1998).

[22] B. B. Blinov et al., Phys. Rev. ST Accel. Beams 2, 064001 (1999).

[23] V.P. Derenchuk and A.S. Belov, in Polarized Gas Targets and Polarized Beams, edited by R. J. Holt and M. A. Miller, AIP Conf. Proc. No. 421 (AIP, New York, 1998), p. 422.

[24] D. L. Friesel and S. Y. Lee, in Proceedings of the 1997 Particle Accelerator Conference, Vancouver, Canada (IEEE, Piscataway, NJ, 1998), p. 935.

[25] This $G \gamma=2$ resonance in CIS was later corrected by the IUCF staff using their new partial Siberian snake. G.W. East, D. L. Friesel, W. T. Sloan, and E. J. Stephenson (private communication). 